\title{
EU PAEDIATRIC INVESTIGATION PLANS (PIPS) MIGHT HARM CHILDREN
}

\author{
K. Rose, T. Benisheva-Dimitrova \\ Faculty of Public Health, Medical University - Sofia, Bulgaria
}

\begin{abstract}
Aim/objective: Since 2007, companies in the EU must submit paediatric investigation plans (PIPS) for new drugs, unless the PIP is waived and the review article investigated if that improve the child healthcare. Methods: We analysed the EU Paediatric Regulation (EUPR), PIP decisions, PIP decision patterns, EU key documents on "better medicines for children" and examined PIP studies versus the epidemiology described in the reference literature. We examined how PIPs translate into studies by checking www. clinicaltrials.gov and www.clinicaltrialsregister.org. We also investigated the medical sense of PIP-demanded clinical studies in adolescents. Results: The EUPR in Art. 2 (1) defines "paediatric population" as those between birth and 18 years. It lists challenges in dosing and safety of drugs in neonates and infants as if these challenges apply to anybody $<18$ years. PIPs demand studies in adolescents although this group needs separate dose finding and efficacy studies only in exceptional cases, if at all. Most PIP studies in rare diseases are unfeasible: too many studies for too few patients in general. Two questionable PIP studies were discontinued in 2016, in one of them several patients died. Conclusions: Neonates and infants have immature organs, with resulting potential for drug over/underdosing. PIPs equalize the legal definition of childhood with a biological limit. The resulting automatism leads to a worldwide threat to children. Most PIP-demanded studies are medically senseless, some even worse. Ethics committees should reject questionable PIP studies and suspend such ongoing studies immediately.
\end{abstract}

Key words: paediatric drug development (PDD), better medicines for children, EU paediatric regulation, paediatric legislation, paediatric investigation plans (PIPS)

Corresponding author: Prof. Tatyana Benisheva, Faculty of Public Health, Medical University, 8 Byalo More Str., 1547 Sofia, tel.: 00359887476909, e-mail: Benicheva_tb@yahoo.com

\section{INTRODUCTION}

ince 2007, new medicinal products need a paediatric investigation plan (PIP) for EU-registration. Without a PIP accepted by the European Medicines Agency (EMA) paediatric committee (PDCO), marketing authorization for adults and children is blocked $[20,23,25]$. The EUPR demands PIPs also for rare diseases, diseases that exist only or predominantly in children, vaccines, and biologics. More than 1000 PIP decisions are listed on the EMA website in January 2017 [9].

Modern pharmaceutical legislation (MPL) was introduced in the US after the thalidomide catastrophe that led to ten thousands of children with malformed extremities in the period 1959-1962 [18, 25]. Following the US precedence, MPL was introduced world- 
wide gradually. MPL resulted in modern labels and protected against misleading claims and quack medicines. Only since modern labels exist, there is the difference between on-label and off-label treatment. Thus, MPL also triggered broad off-label use of drugs in children, as in the 1960 s drugs were routinely licensed to adults first. After the 2nd World War, more and more efficacious drugs became broadly available [18]. MPL reflects that modern medicinal products are not only efficacious but also can have serious adverse reactions and require continuous control before and after approval. MPL ensures that new medicines go through clinical and further trials before they can be prescribed. Regulatory authorities (RAs) became a third key player in medical progress, complementing the medical profession and pharmaceutical industry as drives of medical innovation. When MPL was introduced, clinical trials were mostly performed in young men, often prisoners. Children were perceived as vulnerable beings that needed protection.

In 1963, Shirkey coined the term of children as «therapeutic orphans», reflecting two concerns: legal problems for the medical doctor if something goes wrong with off-label treatment in children, and concerns that children might be treated worse than adults [35].

Since the 1960s, paediatric clinical pharmacology (PCP) showed how immature babies' and infants' organs are, and that mechanical titration of drug doses to their body weight can result in under- or overdosing [19]. Since 1997, the US paediatric legislation (USPL) offered voluntary rewards to industry for studies in minors; later the FDA also could mandate paediatric studies without reward [25]. Already the USPL treated the legal age limit of childhood also as a biologic limit. But age is only a vague indicator of the body's maturity. Absorption, distribution, metabolism, excretion (ADME) of neonates and babies can be very different from school children, adolescences, and adults. Adolescents' bodies (not the brain) become adult before the 18th birthday. The USPL has several restrictions, e.g., orphan diseases are exempt from FDA-ordered paediatric studies, and the FDA can mandate paediatric trials only in the same indication as in adults.

Based on the above explanation, one of the key questions is: can clinical trials demanded in EMAPIPs improve child healthcare?

\section{METHODS}

1. We have analysed the EUPR's wording.

2. We reviewed PIP decisions, published on the EMA website and via googling the respective PIP number.
3. We have investigated the epidemiology of some diseases for which PIPs demand paediatric studies against the data described in the scientific literature.

4. We have analysed how PIPs translate into physical studies by checking PIP-demanded trials against trials listed on www.clinicaltrials.gov and www.clinicaltrialsregister.org. Furthermore, we have analysed their medical sense.

\section{RESULTS}

Article 2 of the EUPR (Table 1) describes testing before approval and Recital 2 declares the forces of the market as insufficient to promote paediatric drug development (PDD).

Recital 3 lists the challenges detected by PCP in newborns and infants, including death as if these challenges exist in everyone $<18$ (Table 1). However, this is not the case. While ADME in preterm neonates and neonates can differ strongly from adults, in adolescents the body matures and usually becomes adult before the 18th birthday. These adolescents are legally still minors, but their body is already mature. For drug treatment, adult doses are medically indicated.

Table 1. EUPR Recitals and Article 2 (1)

\begin{tabular}{|l|l|}
\hline Recital 1 & $\begin{array}{l}\text { Before a medicinal product for human use is } \\
\text { placed on the market in one or more Member } \\
\text { States, it generally has to have undergone exten- } \\
\text { sive studies, including preclinical tests and clini- } \\
\text { cal trials, to ensure that it is safe, of high quality } \\
\text { and effective for use in the target population. }\end{array}$ \\
\hline Recital 2 & $\begin{array}{l}\text { Such studies may not have been undertaken } \\
\text { for use in the paediatric population and many } \\
\text { of the medicinal products currently used to treat } \\
\text { the paediatric population have not been studied } \\
\text { or authorised for such use. Market forces alone } \\
\text { have proven insufficient to stimulate adequate } \\
\text { research into, and the development and authori- } \\
\text { sation of, medicinal products for the paediatric } \\
\text { population. }\end{array}$ \\
\hline Recital 3 & $\begin{array}{l}\text { Problems resulting from the absence of suitably } \\
\text { adapted medicinal products for the paediatric } \\
\text { population include inadequate dosage informa- } \\
\text { tion which leads to increased risks of adverse } \\
\text { reactions including death, ineffective treatment } \\
\text { through under-dosage, non-availability to the } \\
\text { paediatric population of therapeutic advances, } \\
\text { suitable formulations and routes of administra- } \\
\text { tion, as well as use of magistral or officinal for- } \\
\text { mulations to treat the paediatric population which } \\
\text { may be of poor quality. }\end{array}$ \\
\hline Article (1) & $\begin{array}{l}\text { paediatric population' means that part of the } \\
\text { population aged between birth and 18 years; }\end{array}$ \\
\hline
\end{tabular}


The remainder of the EUPR empowers the EMA to create PDCO and PIP system, and further operative consequences. The EUPR does not address who, how or what will be used to evaluate its effectiveness.

The key elements of all PIP decisions are published on the EMA website [9]. Googling the PIP number gives access to the respective PIP decision.

The American Academy of Pediatrics (AAP) has established a clear and pragmatic position on off-label drug use in children [17]. A comparable position statement has not been published by European paediatricians, but would be desirable. Instead, the EMA has adopted a position that characterizes off-label as bad [10] without differentiating between existing dangers of off-label vs. its life-saving efficacy as it has been proven in $\mathrm{PO}[1,25,31,32]$ and neonatology [22].

Four PIPs published on the EMA website demand "paediatric" clinical trials in adolescents between physiologically defined adulthood and the 18th birthday:

- EMEA-001264-PIP01-12 for chondrocytes used endoscopically for repair of damaged joints: the PIP asks for a study between closure of the growth plates and the 18th birthday;

- EMEA-000250-PIP01-08-M02 and EMEA000658-PIP01-09, two contraceptives: clinical trials are demanded to compare pharmacokinetics (PK) among young women after menarche and before the 18th birthday vs. PK in women aged 18 to 50 years;

- EMEA-001492-PIP01-13, trifarotene, an anti-acne medication. The PIP demands two trials (one comparing different trifarotene concentrations to placebo in "children" and adults, the other one comparing trifarotene to the vehicle, again in "children" and adults. "Children" are defined as "from puberty to less than 18 years of age".

In these four examples, the "paediatric" patients or "children" are no longer children. Instead, they are legally minors, but biologically their body is adult, as documented in the inclusion criteria. Medically, adulthood of the reproductive organ system is defined by the end of puberty, and adulthood of the bones by the closure of growth plates at the end of puberty [34].

Table 2 shows 11 PIPs that demand dermatology studies in "children" aged 2-17 or 12-17 years. Even healthy newborns have already a skin whose thickness is comparable to adults, and only preterm newborns have considerably thinner skin [19]. What additional medical information can we expect from such separate dermatology trials in «children» whose skin is similar to the adult, which is the case in 12-17-yearolds, or from trials that demand patients be aged between 2 and 17 years?

While the medical value of PIP-demanded trials in adolescents can be assessed by combining basic knowledge of human physiology and growth with common sense, it is more complicated with PIPs for diseases that are frequent or rare in childhood. A single PIP and the paediatric studies it demands may seem reasonable. However, this changes if the decision patterns across the respective disease area are examined.

Table 2. Dermatology PIP Decisions In Children \& Adolescents

\begin{tabular}{|l|l|l|l|l|}
\hline$\#$ & Compound & Indication & PIP Number & Age \\
\hline 1 & adalimumab & Non-infectious uveitis & EMEA-000366-PIP05-12 & $2-17 \mathrm{y}$ \\
\hline 2 & adalimumab & hidradenitis suppurtiva & EMEA-000366-PIP01-08-M06 & $12-17 \mathrm{y}$ \\
\hline 3 & Bisoctrizole/TD & Solar urticaria & EMEA-000585-PIP01-09 & $12-17 \mathrm{y}$ \\
\hline 4 & Clindamycin/Tretinoin & acne vulgaris & EMEA-000892-PIP01-10 & $12-17 \mathrm{y}$ \\
\hline 5 & Botulinum neurotoxin & muscle spasticity & EMEA-001039-PIP01-10-M01 & $2-17 \mathrm{y}$ \\
\hline 6 & Human immunoglobulin & dermatopolymyositis & EMEA-000415-PIP01-08-M01 & $2-17 \mathrm{y}$ \\
\hline 7 & MAB to IL-31 receptor & atopic dermatitis & EMEA-001624-PIP01-14 & $2-17 \mathrm{y}$ \\
\hline 8 & Methyl aminolevulinate & acne vulgaris & EMEA-000698-PIP02-10-M01 & $>12 \mathrm{y}$ \\
\hline 9 & Nalfurafine hydrochloride & Severe uraemic pruritus & EMEA-000266-PIP01-08-M01 & As vs. ado (12-17 y) \\
\hline 10 & Tazarotene & lamellar ichthyosis & EMEA-000510-PIP02-10-M02 & $2-17 \mathrm{y}$ \\
\hline 11 & Terbinafine hydrochloride & onychomycosis & EMEA-001259-PIP02-13 & $2-17 \mathrm{y}$ \\
\hline
\end{tabular}

Abbreviations: ado - adolescents; As - adults; IL - intrleukin; MAB - monoclonal antibody; TD - titanium dioxide 
Hay fever, frequent in adults and children, can be treated and prevented by specific immunotherapy (SIT), described since 1911 [29]. In the decades thereafter, allergens were increasingly processed industrially but continued in a niche apart from MPL. When Germany introduced a law requiring registration of SIT allergen products as drugs in 2008, PIPs were already mandatory. The German Paul-EhrlichInstitute and the EMA elaborated an allergen standard PIP jointly [29], resulting in >100 PIPs demanding five-year double-blind placebo-controlled clinical studies in children and adolescents. Fifty-eight PIPs with such studies must be executed until 2031, or manufacturers will have to withdraw their products $[8,29]$. SIT is known to be efficacious for more than 100 years. Switzerland simply allowed easy retrograde approval of SIT allergen products. No increased number of dead patients due to not following EU rules has been reported in the Swiss medical literature.

PIPs are mandatory unless the disease is PIP-exempted in the EMA list of class waivers [11]. Originally, melanoma was class waived, but this decision was revoked in 2008, claiming that enough patients exist for clinic studies [12, 13, 25, 32, 33]. The EMA's justification omitted that $3 / 4$ of juvenile melanoma patients are healed by surgical tumor removal without systemic treatment and that the US database used for the EMA's justification groups 15-19 years olds together, of whom the $18 \& 19$ years olds are already adults. Thus, only about $10 \%$ of the patients the EMA claims to exist could theoretically be recruited into «paediatric» melanoma studies. Two PIP-triggered international studies in adolescents with metastasized melanoma had to be terminated in 2016 due to slow recruitment: vemurafenib with 26 sites in the US, EU and Australia [6], and ipilimumab with 30 sites in the US, EU and Mexico [21].

Four more studies continue to recruit minors with melanoma and other solid tumors [2, 3, 4, 36]. And more companies will have to initiate such studies, as they want to authorise medicinal products in the EU [32]. Paediatric studies are reported as "successes" in EMA reports, without differentiating if they make sense or not [15]. We have major doubts if the two melanoma studies that had to be discontinued in 2016 should be regarded as successes.

PIPs demand 15 studies in paediatric multiple sclerosis (pMS), although the International Clinical pMS Group estimates that only 1-2 parallel pMS studies are possible worldwide [7, 30].

PIPs also demand multiple paediatric trials in paediatric psoriasis with modern biologics, which use should be reserved for severe cases of psoriasis which in children is too rare for multiple paediatric studies [28].

PIPs demand numerous paediatric trials for a multitude of tyrosine kinase inhibitors aiming at chronic myelogenous leukaemia (CML) [31].

In 2015, more class waivers were revoked by EMA, including, e.g., for drugs aiming at liver or kidney cancer, which both are extremely rare in minors [13].

\section{DISCUSSION/CONCLUSIONS}

While MPL was triggered worldwide by the thalidomide catastrophe, no comparable catastrophe triggered USPL/EUPL. EU regulators made claims about "lack of availability of appropriate medicines for children" [24] or "neglect of children in the development of effective and safe medicinal products" [20]. But child healthcare has continuously improved since the 2nd World War, including neonatology [22] and paediatric oncology $(\mathrm{PO})[1,25,26]$. PO systematic tested adult chemotherapy compounds in children with cancer. The clinical trials performed by PO were not regulatory trials $[1,26]$.

Paediatric legislation demands paediatric clinical trials in new drugs that are predominantly developed for adult diseases [5]. Since 2007 this has not changed. But we see the result of 10 years EMA activity.

Revoking the melanoma class waiver in 2008 was not a reasonable decision. This will not bring "better medicines for children" [14]. Now EMA/PDCO think that by intensification of the PIP system things will improve [13]. Is this the right way? Based on the PIP analysis for melanoma no additional clinical benefit for children with even rarer diseases can be expected by the expansion of the PIP system. What benefit can be expected from PIPs for various new compounds, e.g., liver cancer? It will only intensify the search for the rare children with liver cancer, without helping them. Of course, such studies will be reported by the EMA as "successes" - every EMA report so far has reported "more" paediatric studies as successes [15].

Drug development is expensive, controversial and complex [33]. Initial enthusiasm over new efficacious drugs, including antibiotics, steroids, and betablockers has given place to a more critical view of drug development [18]. PCP has shown the potential for over- and underdosing in babies and infants [19]. With the EUPR the EU wanted to outperform the original USPL. By removing all caveats, it results in demands for clinical studies in everybody under 18 irrespective if these make medical sense or are 
feasible. However, children do not need more clinical studies, they need reasonable studies.

Is the paediatric labelling of the first recombinant asparaginase for the treatment of acute lymphoblastic leukaemia (ALL), reported as a major success by the EMA [15, p.14], a real success? Among all types of child malignoma, ALL is the best success story of PO, with survival rates already approaching $90 \%$. There remains a high medical need for better treatment of children with ALL indeed. Still, around 10\% do not survive, and the survivors struggle all their life with the consequences of the toxic, albeit lifesaving treatment. Children with ALL do need new treatment modalities. Compared to these real medical needs, the medical value of paediatric labelling of asparaginase is almost negligible, and certainly not a major success. Medical and pharmaceutical innovation in PO will not be achieved by enforcing paediatric labelling of new pharmaceutical forms of cytotoxic compounds. Children with cancer need new innovative medicines, not an abuse of children in multiple paediatric studies that later have to be terminated because they are unfeasible. How many more children with melanoma and other rare types of cancer (almost all cancer types in children are rare) must die in unfeasible trials whose rationale is not based on science?

Few will today object to a statement like "Children and infants deserve the same right to treatment as adults" which was written in 1999 [37]. Many attitudes have changed since the 1960s MPL was introduced. MPL had introduced minimal control over compounds that before could become broadly available with potentially catastrophic consequences. Equalization of legal age definition of adulthood with biological maturation reflects short-sightedness at the interface of medicine and law and leads to medical errors. Eventually, this will need tackling from two sides: (1) adolescents whose bodies become adult before legal adulthood, and (2) babies and infants.

Expanding drug labels from adults to adolescents might require a separate law that would allow extrapolation of clinical trials data from adults to adolescents and prescription of these medicines without extra trials. Modelling \& simulation (M\&S), followed by PK/PD dose confirmation in small paediatric trials, allow dose estimation in children down to two years of age. Automatic demand for separate safety \& efficacy trials in the 2-17 years population for all diseases is not based on science.

In all diseases that are rare in childhood but for which drugs are developed for adults, PIPs demand paediatric studies. The PIP system disregards whether studies are feasible or not. The number of demanded studies in children makes them unfeasible altogether, as is shown in this review. Ethics committees (ECs) are not yet aware of this challenge, but they will have to. All ongoing trials are listed in www.clinicaltrials. gov and www.clinictrialsregister.eu and can be easily consulted. Once IRBs/ECs begin realizing the potential threat of PIPs for children, they will check submitted paediatric protocols against ongoing trials that compete for the same study population. ECs should reject questionable PIP-demanded paediatric studies and suspend those ongoing.

It is bizarre that now pharmaceutical companies and clinical research organisations (CROs) must protect children against EU regulators which demands unfeasible clinical trials. They will need help from ECs. The EUPR needs fundamental modification.

Some initiatives, e.g., the US Creating Hope Act, facilitate truly new drug development for rare paediatric and adult diseases [16]. Adults and children in the EU need more such facilitation as well [27].

\section{REFERENCES}

1. Adamson PC. Improving the outcome for children with cancer: development of targeted new agents. CA Cancer J Clin. 2015;65: 212-220.

2. A Phase I/II, Multicenter, Open-Label, Dose-Escalation Study Of The Safety And Pharmacokinetics Of Cobimetinib In Pediatric And Young Adult Patients With Previously Treated Solid Tumors. https://www.clinicaltrialsregister.eu/ctr-search/ search?query=2014-004685-25 Accessed 20JAN2017.

3. A Study of Pembrolizumab (MK-3475) in Pediatric Participants With Advanced Melanoma or Advanced, Relapsed, or Refractory PD-L1-Positive Solid Tumors or Lymphoma (MK3475-051/KEYNOTE-051). https://clinicaltrials.gov/ct2/show/ NCT02332668 (Accessed 20JAN2017).

4. A Study to Determine Safety, Tolerability and Pharmacokinetics of Oral Dabrafenib In Children and Adolescent Subjects. https://clinicaltrials.gov/ct2/show/NCT01677741 (Accessed 20JAN2017).

5. Boots I, Sukhai RN, Klein RH, et al. Stimulation programs for pediatric drug research - do children really benefit? Eur J Pediatr. 2007; 166: 849-855.

6. BRIM-P. A study of Vemurafenib in pediatric patients with stage IIIC or stage IV melanoma harboring BRAFV600 mutations. https://clinicaltrials.gov/ct2/show/NCT01519323 (Accessed 20JAN2017).

7. Chitnis T, Tenembaum S, Banwell B et al. Consensus statement: evaluation of new and existing therapeutics for pediatric multiple sclerosis. Mult Scler. 2012 Jan;18(1):116-27

8. Eichler I, Sala Soriano E. Close collaboration between academia, industry and drug regulators is required in the development of allergen products for specific immunotherapy in children. Allergy 2011: 66: 999-1004. 
9. EMA website. www.ema.europa.eu (Accessed 20JAN2017)

10. EMA 2004: Evidence of harm from off-label or unlicensed medicines in children. www.ema.europa.eu/docs/en_GB/ document_library/Other/2009/10/WC500004021.pdf

11. EMA 2011. EMA class waivers, http://www.ema.europa.eu/docs/en GB/document library/Other/2011/12/ WC500119981.pdf (Accessed 20FEB2017).

12. EMA 2010. European Medicines Agency decision $P / 345 / 2010$ of 20 December 2010 on a class waiver on condition(s) in accordance with Regulation (EC) No 1901/2006 of the European Parliament and of the Council. http://www.ema. europa.eu/docs/en_GB/document_library/Other/2009/11/ WC500011500.pdf. Accessed 20JAN2017 (Accessed 20JAN2017).

13. EMA 2015. European Medicines Agency decision CW/0001/2015 of 23 July 2015 on class waivers, in accordance with Regulation (EC) No 1901/2006 of the European Parliament and of the Council. www.ema.europa.eu/docs/ en_GB/document_library/Other/2015/07/WC500190385.pdf

14. EMA 2015. Better Medicines for Children. www.ema.europa.eu/docs/en_GB/document_library/Leaflet/2009/12/ WC500026493.pdf

15. EMA2016. 10-year Report to the European Commission. General report on the experience acquired as a result of the application of the Paediatric Regulation. http://ec.europa. eu/health//sites/health/files/files/paediatrics/2016_pc_report_2017/ema_10_year_report_for_consultation.pdf. Accessed 20JAN2017 (Accessed 20JAN2017).

16. FDA. Best Pharmaceuticals for Children Act and Pediatric Research Equity Act July 2016. Status Report to Congress. www.fda.gov/downloads/ScienceResearch/SpecialTopics/ PediatricTherapeuticsResearch/UCM509815.pdf (Accessed 20JAN2017).

17. Frattarelli DA, Galinkin JL, Green TP et al. Off-label Use of Drugs in Children. Pediatrics 2014, 133 (3), 563-567.

18. Hilts PJ. Protecting Americass Health: The FDA, Business, and One Hundred Years of Regulation. University of North Carolina Press 2004.

19. Kearns GL, Abdel-Rahman SM, Alander SW et al. Developmental Pharmacology - Drug Disposition, Action, and Therapy in Infants and Children. N Engl J Med. 2003;349(12):1157-1167.

20. Mentzer D. Progress review of the European Paediatric Regulatory Framework after six years of implementation. Int J Pharm. 2014 Aug 5;469(2):240-3.

21. Phase 2 study of Ipilimumab in children and adolescents (12 to $<18$ Years) with previously treated or untreated, unresectable stage III or stage IV malignant melanoma. https://clinicaltrials.gov/ct2/ show/NCT01696045.

22. Philip AG. The Evolution of Neonatology. Pediatr Res. 2005 Oct;58(4):799-815.

23. Regulation (EC) No 1901/2006 Of The European Parliament And Of The Council of 12 December 2006 on medicinal prod- ucts for paediatric use and amending Regulation (EEC) No 1768/92, Directive 2001/20/EC, Directive 2001/83/EC and Regulation (EC) No 726/2004. Official Journal of the European Union, 27.12.2006, L 378/1 - L 278/19 http://ec.europa.eu/ health/files/eudralex/vol-1/reg_2006_1901/reg_2006_1901_ en.pdf pdf (Accessed 20JAN2017).

24. Rocchi F, Paolucci P, Ceci A, Rossi P. The European paediatric legislation: benefits and perspectives. Italian Journal of Pediatrics 2010, 36:56 Link: http://www.ncbi.nlm.nih.gov/ pmc/articles/PMC2933611/pdf/1824-7288-36-56.pdf (Accessed 20JAN2017).

25. Rose K. Pediatric Pharmaceutical Legislation in the USA and EU and Their Impact on Adult and Pediatric Drug Development. In: Bar-Shalom D \& Rose K: Pediatric Formulations - A Roadmap, AAPS \& Springer, 2014: chapter 28, pp. 405-420.

26. Rose K. European Union Pediatric Legislation Jeopardizes Worldwide, Timely Future Advances in the Care of Children With Cancer. Clinical Therapeutics 2014, 36 (2), 163-177.

27. Rose K. New Drugs For Rare Diseases in Children. Clin Ther 2017 , in press.

28. Rose K \& Happle R. The Impact of Regulation on Pediatric Psoriasis Drug Approvals: The Challenge of the European Union (EU) Pediatric Investigation Plans. Pediatric Dermatology 2017 , in press.

29. Rose K \& Kopp MV. Pediatric investigation plans for specific immunotherapy: Questionable contributions to childhood health. Pediatr Allergy Immunol. 2015 Dec;26(8):695-701.

30. Rose K \& Mueller T. Children with Multiple Sclerosis Should Not Become Therapeutic Hostages. Ther Adv Neurol Disord 2016, 9(5) 389-395.

31. Rose K \& Walson PD. The contributions of the European Medicines Agency and its pediatric committee to the fight against childhood leukemia. Risk Manag Healthc Policy. 2015 Nov 5;8:185-205.

32. Rose K \& Walson PD. Do the European Medicines Agency (EMA) Decisions Hurt Pediatric Melanoma Patients? Clin Ther 2017, in press.

33. Rose K \& Senn S. Drug development: EU paediatric legislation, the European Medicines Agency and its Paediatric Committee - adolescents' melanoma as a paradigm. Pharmaceutical Statistics 2014; 13(4): 211-213.

34. Shim KS. Pubertal growth and epiphyseal fusion. Ann Pediatr Endocrinol Metab. 2015 Mar;20(1):8-12.

35. Shirkey H. Therapeutic Orphans. Pediatrics 1999 Sep;104(3 Pt 2):583-584.

36. To Find a Safe Dose and Show Early Clinical Activity of Weekly Nab-paclitaxel in Pediatric Patients With Recurrent/ Refractory Solid Tumors https://clinicaltrials.gov/ct2/show/ NCT01962103 (Accessed 20JAN2017).

37. Turner S, Nunn AJ, Fielding K, Choonara I. Adverse drug reactions to unlicensed and off-label drugs on paediatric wards. Acta Paediatr 1999, 88: 965-968. 